\title{
Scaling properties at the interface between different critical subsystems: The Ashkin-Teller model
}

\author{
Péter Lajkó, ${ }^{1}$ Loïc Turban, ${ }^{2, \text { * }}$ and Ferenc Iglói ${ }^{3,4}$, 团 \\ ${ }^{1}$ Department of Physics, Kuwait University, P.O. Box 5969, Safat 13060, Kuwait \\ ${ }^{2}$ Laboratoire de Physique des Matériaux, Université Henri Poincaré, BP 239, \\ F-54506 Vandouvre lès Nancy Cedex, France \\ ${ }^{3}$ Research Institute for Solid State Physics and Optics, H-1525 Budapest, P.O.Box 49, Hungary \\ ${ }^{4}$ Institute of Theoretical Physics, Szeged University, H-6720 Szeged, Hungary
}

(Dated: November 30, 2018)

\begin{abstract}
We consider two critical semi-infinite subsystems with different critical exponents and couple them through their surfaces. The critical behavior at the interface, influenced by the critical fluctuations of the two subsystems, can be quite rich. In order to examine the various possibilities, we study a system composed of two coupled Ashkin-Teller models with different four-spin couplings $\epsilon$, on the two sides of the junction. By varying $\epsilon$, some bulk and surface critical exponents of the two subsystems are continuously modified, which in turn changes the interface critical behavior. In particular we study the marginal situation, for which magnetic critical exponents at the interface vary continuously with the strength of the interaction parameter. The behavior expected from scaling arguments is checked by density matrix renormalization group calculations.
\end{abstract}

\section{INTRODUCTION}

Realistic systems have a finite extent and, when they display a second-order phase transition, the critical behavior in the boundary region is generally different from that in the bulk. The characteristic size of this region is given by the correlation length, which becomes divergent as the critical temperature $T_{c}$ is approached. In the vicinity of the critical point, the singularities of local quantities, such as the surface magnetization, are characterized by critical exponents, which are generally different from their bulk counterparts. This type of local critical phenomena has been thoroughly studied in the case of a free surface through exact, field-theoretical and numerical methods, $1,2,3$

When a system is in contact through its boundary with another system, the environment can influence the local critical behavior at the interface. If, however, the critical temperature of the environment, $T_{c}^{\prime}$, is different from $T_{c}$, the nature of the transitions at the interface is expected to be the same as for a surface. $\underline{\underline{4}}$ If the environment has the higher critical temperature $T_{c}^{\prime}>T_{c}$, it stays ordered at $T_{c}$ and the interface transition has the same properties as the extraordinary surface transition., ${ }^{1,2,3}$ In the opposite case, for $T_{c}^{\prime}<T_{c}$, the environment is disordered at $T_{c}$ and the interface transition is actually an ordinary surface transition. $1,2,3$

Here we consider the more complex problem when the two subsystems in contact have the same critical temperature but not the same set of critical exponents. Thus, the competition between the two different bulk and surface critical behaviors may result in a completely new type of interface critical phenomena. This problem has already been addressed in Ref. 5 in which the analytical mean-field solution, in terms of $\varphi^{k}$ field theories, has been obtained and generalized by using phenomenological scaling considerations. Monte Carlo simulations have also been performed in two dimensions for interfaces between subsystems belonging to the universality classes of the Ising model, the three-state and four-state Potts models.

In all these examples the stable fixed points are related to surface critical behavior and the expected renormalization group ( $\mathrm{RG}$ ) phase diagram is the one given in the upper part of Fig. 2] For weak interface couplings, the junction renormalizes to a cut, and we have the same local critical behavior as for a free surface, whereas for strong couplings, the interface becomes ordered at the bulk transition temperature. For some intermediate value of the couplings, there is a special interface transition fixed point, involving new critical exponents, which, however, can be expressed in terms of the bulk and surface exponents of the two subsystems. $\frac{5}{5}$

In the present work our purpose is to examine the different types of possible interface critical behavior which can be realized. Thus, we consider situations where a weak interface coupling can be irrelevant, relevant or even truly marginal. We are particularly interested in the latter case. A convenient system, for which all these different situations can be realized, is the two-dimensional (2D) Ashkin-Teller (AT) model,, 6 or its one-dimensional (1D) quantum version. 7,8

By introducing two Ising variables per site, the AT Hamiltonian can be rewritten as two Ising Hamiltonians coupled through a four-spin interaction, $\frac{9}{-}$ which is a truly marginal operator. As a consequence, some bulk and surface critical exponents are continuously varying functions of the strength of the four-spin coupling $\epsilon$. These critical exponents are known exactly through conformal invariance ${ }^{10}$ and Coulomb-gas mapping. 11

The composite system which we consider consists of two AT models with the same critical temperature but different four-spin couplings, and thus different sets of critical exponents. We couple these subsystems through their surface spins and study the critical properties at 
the interface while varying the strength of the interface coupling. We first classify the possible interface critical behaviors through scaling considerations, which are then confronted with the results of extensive numerical calculations using the density matrix renormalization group (DMRG).

The structure of the paper is as follows. The AT model and its basic critical properties are described in Sec. [II] We define the composite system and discuss its possible interface RG phase diagrams in Sec. III] Results of numerical calculations are presented in Sec. IV and discussed in Sec. V.

\section{ASHKIN-TELLER MODEL AND ITS CRITICAL PROPERTIES}

The AT model is defined in terms of two sets of Ising spin variables $\sigma_{i}= \pm 1$ and $\tau_{i}= \pm 1$, attached to each lattice site $i$. The usual Ising interaction $K\left(\sigma_{i} \sigma_{j}+\tau_{i} \tau_{j}\right)$ between nearest-neighbor sites $i$ and $j$ is supplemented by a four-spin interaction $K_{4} \sigma_{i} \sigma_{j} \tau_{i} \tau_{j}$, which is parametrized as $K_{4}=K \epsilon$. This latter term represents the product of the energy densities in the two Ising systems. We consider the system on a square lattice and work with the row-to-row transfer matrix $\mathcal{T}$. In the Hamiltonian limit, the transfer matrix can be written as $\mathcal{T} \sim \exp \left(-\kappa \mathcal{H}_{A T}\right)$, where $\kappa$ is the lattice spacing in the "time" direction and $\mathcal{H}_{A T}$ is the $1 \mathrm{D}$ quantum Hamiltonian given by

$$
\begin{aligned}
\mathcal{H}_{A T}= & -\sum_{i=1}^{L-1}\left(\sigma_{i}^{z} \sigma_{i+1}^{z}+\tau_{i}^{z} \tau_{i+1}^{z}\right)-h \sum_{i=1}^{L}\left(\sigma_{i}^{x}+\tau_{i}^{x}\right) \\
& -\epsilon\left[\sum_{i=1}^{L-1} \sigma_{i}^{z} \sigma_{i+1}^{z} \tau_{i}^{z} \tau_{i+1}^{z}+h \sum_{i=1}^{L} \sigma_{i}^{x} \tau_{i}^{x}\right] .
\end{aligned}
$$

Here, $\sigma_{i}^{x, z}$ and $\tau_{i}^{x, z}$ are two sets of Pauli matrices and $h$ is the strength of the transverse field, which plays the role of the temperature in the classical system. One can introduce a set of dual Pauli operators $\widetilde{\sigma}_{i+1 / 2}^{x, z}$ and $\widetilde{\tau}_{i+1 / 2}^{x, z}$ such that

$$
\begin{aligned}
\tilde{\sigma}_{i+1 / 2}^{x}=\sigma_{i}^{z} \sigma_{i+1}^{z}, & \sigma_{i}^{x}=\widetilde{\sigma}_{i-1 / 2}^{z} \widetilde{\sigma}_{i+1 / 2}^{z} \\
\widetilde{\tau}_{i+1 / 2}^{x}=\tau_{i}^{z} \tau_{i+1}^{z}, & \tau_{i}^{x}=\widetilde{\tau}_{i-1 / 2}^{z} \widetilde{\tau}_{i+1 / 2}^{z} .
\end{aligned}
$$

When the Hamiltonian in Eq. (11) is rewritten in terms of the dual variables, the couplings $J_{i}=1$ and the transverse fields $h_{i}=h$ exchange their roles. Consequently, the homogeneous system is self-dual and the self-duality line is located at $h_{c}=1$. For $-1 / \sqrt{2} \leq \epsilon \leq 1$ this is just the critical line separating the ferromagnetic and the paramagnetic phases of the system. In the region $-1<\epsilon \leq-1 / \sqrt{2}$, for $h_{c}^{-}(\epsilon)<h<h_{c}^{+}(\epsilon)$, there is a so-called critical fan in which the system stays critical. ${ }^{7}$ At the critical point, the excitation energy $\Delta E$ and the wave vector $k$ are linearly related, $\Delta E=v_{s} k$, and the sound velocity is given by $\underline{12}$

$$
v_{s}=\frac{\pi \sin (\arccos \epsilon)}{\arccos \epsilon}
$$

In the critical system, the basic operators are the magnetization $\mathcal{O}_{m}(i)=\sigma_{i}^{z}\left(\tau_{i}^{z}\right)$, the energy density $\mathcal{O}_{e}(i)=\sigma_{i}^{z} \sigma_{i+1}^{z}\left(\tau_{i}^{z} \tau_{i+1}^{z}\right)$ or, through duality, $\sigma_{i}^{x}\left(\tau_{i}^{x}\right)$, and the polarization $\mathcal{O}_{p}(i)=\sigma_{i}^{z} \tau_{i}^{z}$. The connected critical correlation functions display a power-law decay, so that $\left\langle\mathcal{O}_{\alpha}(i) \mathcal{O}_{\alpha}(i+r)\right\rangle-\left\langle\mathcal{O}_{\alpha}(i)\right\rangle\left\langle\mathcal{O}_{\alpha}(i+r)\right\rangle \sim r^{-2 x_{\alpha}}$, where $x_{\alpha}$ is the anomalous dimension of $\mathcal{O}_{\alpha}$. Similarly, surface-to-surface correlations involve the corresponding surface dimensions $x_{\alpha}^{s}$.

The critical properties of the AT model are exactly known through conformal invariance and Coulomb-gas mapping. The anomalous dimensions of bulk operators are given by $\underline{\underline{7}}$

$$
x_{m}=\frac{1}{8}, \quad x_{e}=\frac{\pi}{2 \arccos (-\epsilon)}, \quad x_{p}=\frac{1}{4} x_{e} .
$$

The correlation length critical exponent is related to the dimension of the energy density by $\nu=1 /\left(2-x_{e}\right)$ when $-1 / \sqrt{2} \leq \epsilon \leq 1$, whereas it is formally infinite in the critical fan. True marginal behavior implies that the scaling dimension of the operator $\mathcal{O}_{4}(i)$ associated with the fourspin interaction $\sigma_{i}^{z} \sigma_{i+1}^{z} \tau_{i}^{z} \tau_{i+1}^{z}$ keeps the constant value $x_{4}=2$, the same as for the two decoupled Ising chains.

The corresponding anomalous dimensions for surface operators are $\underline{\underline{13}}$

$$
x_{m}^{s}=\frac{\arccos (-\epsilon)}{\pi}, \quad x_{e}^{s}=2, \quad x_{p}^{s}=1 .
$$

One may notice that the anomalous dimensions, which are $\epsilon$ dependent in the bulk due to the presence of the marginal four-spin interactions, remain constant at the surface and vice versa.

\section{COMPOSITE SYSTEM AND RENORMALIZATION GROUP PHASE DIAGRAMS}

\section{A. Ladder and chain junctions}

A composite AT system is obtained by coupling two different semi-infinite subsystems through their surface spins. These subsystems have the same nearest-neighbor coupling, thus the same critical temperature. They have different values of the four-spin couplings $\epsilon^{(-)}\left(\epsilon^{(+)}\right)$for $z<0(z>0)$ with $\epsilon^{(-)} \leq \epsilon^{(+)}$.

The junction can be of two different kinds, ladder or chain junction ${ }^{14}$ (see Fig. 6.1 of Ref. 15). In the ladder junction, there are nearest-neighbor as well as four-spin couplings between sites at $i=-1$ (boundary of the $z<0$ subsystem) and $i=1$ (boundary of the $z>0$ subsystem). In the Hamiltonian limit, this corresponds to a term

$$
\mathcal{V}_{-1,1}=-J\left(\sigma_{-1}^{z} \sigma_{1}^{z}+\tau_{-1}^{z} \tau_{1}^{z}+\epsilon_{\mathrm{int}} \sigma_{-1}^{z} \sigma_{1}^{z} \tau_{-1}^{z} \tau_{1}^{z}\right),
$$

and the complete Hamiltonian is written as:

$$
\mathcal{H}=\mathcal{H}_{A T}^{(-)}+\mathcal{H}_{A T}^{(+)}+\mathcal{V}_{-1,1} .
$$


In the case of the chain junction, we introduce an extra line of spins at $z=0$, which are connected horizontally to the two subsystems through the respective bulk couplings and there is a two-spin interaction associated with the junction in the vertical direction. In the Hamiltonian limit, the different terms in $\mathcal{H}_{A T}^{( \pm)}$are extended up to $i=0$ and the junction involves a transverse-field term

$$
\widetilde{\mathcal{V}}_{0}=-\widetilde{h}\left(\sigma_{0}^{x}+\tau_{0}^{x}+\epsilon_{\text {int }} \sigma_{0}^{x} \tau_{0}^{x}\right) .
$$

The ladder and chain defects are transformed into each other through duality. In the following, we study the ladder problem as defined in Eq. (77).

\section{B. Basic quantities}

\section{Matrix elements}

We are interested in the local critical behavior of the system; in particular, we want to determine the anomalous dimensions associated with the interface, $x_{m}^{\text {int }}$ for the magnetization density $\mathcal{O}_{m}(0)$ and $x_{e}^{\text {int }}$ for the energy density $\mathcal{O}_{e}(0)$. These can be deduced from the finite-size scaling of the singular part of the corresponding matrix elements:

$$
\begin{aligned}
m_{\text {int }}(L) & =\left\langle 0\left|\sigma^{z}( \pm 1)\right| 0\right\rangle \sim L^{-x_{m}^{\mathrm{int}}} \\
e_{\mathrm{int}}^{x}(L) & =\left\langle 0\left|\sigma^{x}( \pm 1)\right| 0\right\rangle-e_{\mathrm{int}}^{x} \sim L^{-x_{e}^{\mathrm{int}}}, \\
e_{\mathrm{int}}^{z}(L) & =\left\langle 0\left|\sigma^{z}(-1) \sigma^{z}(1)\right| 0\right\rangle-e_{\mathrm{int}}^{z} \sim L^{-x_{e}^{\mathrm{int}}} .
\end{aligned}
$$

For the magnetization density, symmetry-breaking boundary conditions are needed. $|0\rangle$ is the ground state of the Hamiltonian in Eq. (77) and $e_{\text {int }}^{x, z}$ is the limiting value of the interface energy density in the infinite system. We note that the two first matrix elements can be calculated on each side of the interface and there are two possible definitions for the energy density, $e_{\mathrm{int}}^{x}$ and $e_{\mathrm{int}}^{z}$, corresponding to vertical and horizontal bonds in the classical model.

\section{Gaps}

These exponents can also be obtained by using conformal invariance $\underline{10}$ The classical system composed of two semi-infinite planes coupled by one junction is mapped through the logarithmic transformation into two infinite strips, each with a width $L / 2$, coupled together by two parallel junctions at their boundaries and thus building a cylinder. In the extreme anisotropic limit, a Hamiltonian $\mathcal{H}_{\text {cyl }}$, similar to $\mathcal{H}$ in Eq. (7), is associated to the transfer matrix along the cylinder but with two junctions and periodic boundary conditions. For a ladder defect, the two junctions are of the form given in Eq. (6), the first between sites $i=-1$ and $i=1$ and the second between sites $i=-L / 2$ and $i=L / 2$. For a chain junction, the two junctions are of the form given in Eq. (8) and placed at $i=0$ and $i=L / 2$.

In the cylinder geometry, the first gap of $\mathcal{H}_{\text {cyl }}$ scales as $1 / L$ for a critical system, and the prefactor is proportional to the anomalous dimension of the magnetization at the junction 16

$$
E_{1}-E_{0}=\frac{2 \pi v_{s}}{L} x_{m}^{\mathrm{int}} .
$$

Other local exponents are similarly related to higher gaps.

Before calculating the anomalous dimensions numerically, we first consider the possible phase diagrams by studying the stability of the different fixed points.

\section{Two identical subsystems}

We start with the symmetrical model where $\epsilon^{(-)}=$ $\epsilon^{(+)}=\epsilon$. In this case there are three fixed points, located at $J=0, J=1$ and $J=\infty$, and corresponding respectively to two disjoint semi-infinite systems (ordinary interface transition), to the homogeneous system (bulk transition), and to a system with an ordered interface (extraordinary interface transition),$\underline{17,18,19}$

\section{Ordinary interface fixed point}

At the ordinary interface fixed point the perturbation takes the form $J \mathcal{O}_{m}(-1) \mathcal{O}_{m}(1)+J \epsilon_{\text {int }} \mathcal{O}_{p}(-1) \mathcal{O}_{p}(1)$. The first operator, involving the product of two surface magnetization operators, has the dimension

$$
x_{\mathrm{int}}=x_{m}^{(-)}+x_{m}^{(+)}=2 x_{m}^{s},
$$

and, thus, the scaling exponent of $J$ is

$$
y_{\mathrm{int}}=d_{\mathrm{int}}-x_{\mathrm{int}}=1-2 x_{m}^{s},
$$

where $d_{\text {int }}=d-1$ is the dimension of the interface. This type of perturbation is irrelevant for $y_{\mathrm{int}}<0$, i.e., for $x_{m}^{s}>1 / 2$, which happens for $\epsilon>0$, whereas it is relevant for $\epsilon<0$. The marginality condition is satisfied for $\epsilon=0$, which is the Ising limit. The second operator, containing the product of two surface polarization operators, has the dimension $\widetilde{x}_{\text {int }}=2 x_{p}^{s}=2$; therefore, this perturbation is always irrelevant.

\section{Bulk fixed point}

The perturbation to the bulk fixed point introduced by the junction now takes the form $\Delta \mathcal{O}_{e}(-1)+\widetilde{\Delta} \mathcal{O}_{4}(-1)$ where $\Delta=J-1$ and $\widetilde{\Delta}=J \epsilon_{\text {int }}-\epsilon$. The dimension of the first operator is $x_{\mathrm{int}}=x_{e}$; thus, the scaling dimension of $\Delta$ is

$$
y_{\mathrm{int}}=d_{\mathrm{int}}-x_{e}=d-1-x_{e}=\nu^{-1}-1 .
$$



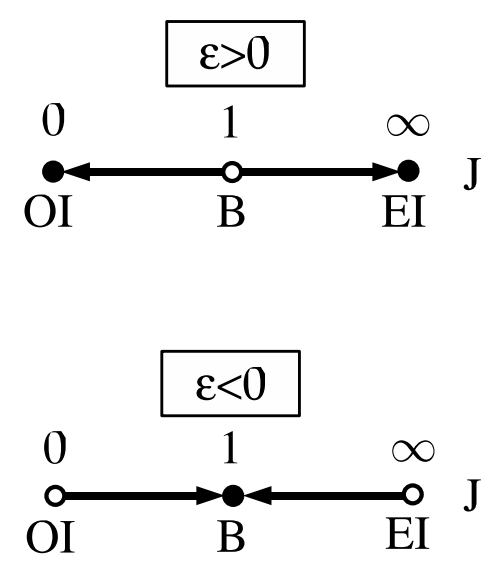

FIG. 1: Schematic RG phase diagram at a ladder defect with coupling $J$ in the critical AT model. The RG flow is different for different signs of the bulk four-spin coupling $\epsilon$. When $\epsilon>0$, the bulk fixed point (B) is unstable; the flow is toward the ordinary interface fixed point (OI) when $J<1$ and the extraordinary interface fixed point (EI) when $J>1$. When $\epsilon<0$, the flow is reversed and the bulk fixed point is always stable for $0<J<\infty$.

This perturbation is relevant (irrelevant) for $\nu<1$ $(\nu>1)$, i.e., for $\epsilon>0(\epsilon<0)$. The marginal situation corresponds once more to the Ising limit $\epsilon=0$. The second operator $\mathcal{O}_{4}$ has the scaling dimension $x_{4}=2$. It follows that $\widetilde{\Delta}$ has the scaling dimension $\widetilde{y}_{\text {int }}=-1$. Thus, the four-spin interface perturbation is always irrelevant as for the ordinary interface fixed point.

\section{Extraordinary interface fixed point}

The stability of this fixed point is related to that of the ordinary interface fixed point. Let us consider the chain junction in Eq. (8). The ordered interface can be realized by setting the transverse field at the fixed point value $\widetilde{h}=0$. Under the duality transformation in Eq. (2), the (weak) chain junction is transformed into a (weak) ladder junction; consequently, to decide about the stability of the corresponding fixed point, one can repeat the argument of Sec. 【IIC1.

\section{Renormalization group phase diagram}

Based on the stability analysis of the fixed points, the expected interface RG phase diagram is depicted in Fig. [1]

When $\epsilon<0$ and for any interface coupling $0<J<\infty$, the behavior at the interface is expected to be governed by the bulk fixed point. Then, the first gap in the spectrum of the conformal Hamiltonian $\mathcal{H}_{\text {cyl }}$ has a $1 / L$ dependence with a prefactor which, according to the gapexponent relation in Eq. (10), is proportional to $x_{m}$.
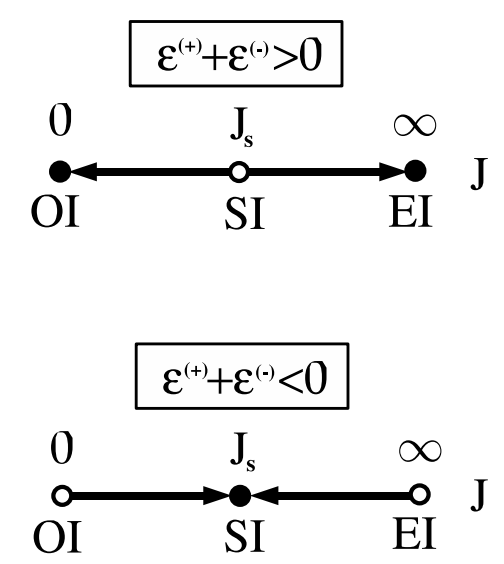

FIG. 2: Schematic RG phase diagram at the interface between two different critical semi-infinite AT models with four-spin couplings $\epsilon^{(-)}<\epsilon^{(+)}$. The interface coupling $J$ is of the ladder type. The direction of the $\mathrm{RG}$ flow depends on the sign of $\epsilon^{(-)}+\epsilon^{(+)}$. When $\epsilon^{(-)}+\epsilon^{(+)}>0$, the special interface fixed point (SI) is unstable; the flow is toward the ordinary interface fixed point (OI) when $J<J_{s}$ and the extraordinary interface fixed point (EI) when $J>J_{s}$. When $\epsilon^{(-)}+\epsilon^{(+)}<0$, the flow is reversed and the special interface fixed point is always stable for $0<J<\infty$.

On the contrary, for $\epsilon>0$ the bulk fixed point is unstable. For weak couplings, $J<1$, the interface renormalizes to a cut and the critical behavior is the same as at a free surface. The first gap in the spectrum of $\mathcal{H}_{\text {cyl }}$ for small $J$ can be estimated perturbatively, as in Sec. IIIC 1, It behaves as the product of the two surface magnetizations, vanishing as $\sim L^{-2 x_{m}^{s}}$, which is faster than $1 / L$ since $x_{m}^{s}>1 / 2$ according to Eq. (5). This indicates that the system is asymptotically breaking into two pieces. For strong couplings $J>1$, the interface remains ordered at the critical temperature and, through duality, the gap has also the size dependence $\sim L^{-2 x_{m}^{s}}$, which is faster than $1 / L$. It corresponds to a vanishing amplitude in Eq. (10) and, thus, to a vanishing interface magnetic exponent, a value which is linked with the local order at the critical point.

In the limit $\epsilon=0$, i.e., when the AT model becomes a system of two noninteracting Ising models, the interface coupling $J$ is a marginal perturbation and the local magnetization exponent is $J$ dependent, $\stackrel{14}{ }$

$$
x_{m}^{\text {int }}(J)=\frac{2}{\pi^{2}} \arctan ^{2}(1 / J), \quad x_{e}^{\text {int }}=1, \quad \epsilon=0 .
$$

Similarly, for a chain junction, $\underline{\underline{14}}$ the local magnetization exponent is $\widetilde{h}$ dependent,

$$
x_{m}^{\mathrm{int}}(\widetilde{h})=\frac{2}{\pi^{2}} \arctan ^{2}(\widetilde{h}), \quad x_{e}^{\mathrm{int}}=1, \quad \epsilon=0 .
$$

The marginal operator is the local energy density, which keeps its anomalous dimension $x_{e}^{\text {int }}=1$, independently of the value of $J$ or $\widetilde{h}$. We note that nonuniversal interface 
critical behavior at a defect plane can be found in the three-dimensional $n$-vector model in the limit $n \rightarrow \infty$, which has been explicitly calculated, 20

\section{Two different subsystems}

If the two subsystems have different four-spin couplings $\epsilon^{(-)}<\epsilon^{(+)}$, one can no longer define a bulk system fixed point. However, the ordinary and extraordinary interface fixed points still exist. The stability analysis of the ordinary interface fixed point can be performed along the lines of Sec III C1 leading to an interface exponent $y_{i}=1-x_{m}^{(-)}-x_{m}^{(+)}$. The ladder perturbation is irrelevant, i.e., the ordinary interface fixed point is stable (unstable) for $\epsilon^{(-)}+\epsilon^{(+)}>0(<0)$. Through duality, as described in Sec II C3. the same type of stability is expected to hold for the extraordinary interface fixed point, too. Consequently, the directions of the RG flows are analogous to the case of identical subsystems in Fig. 1] just the role of the bulk fixed point is taken over by a new special interface fixed point, located at $J_{s}=O(1)$, which controls a special transition. The expected RG phase diagram is given in Fig. 2,

The stability or instability of the special interface fixed point requires that the scaling dimension of the local energy-density operator satisfies

$$
\begin{gathered}
x_{e}^{\text {int }}>1 \text { for } \epsilon^{(-)}+\epsilon^{(+)}<0, \\
x_{e}^{\text {int }}<1 \text { for } \epsilon^{(-)}+\epsilon^{(+)}>0
\end{gathered}
$$

at this fixed point.

In the borderline case, $\epsilon^{(-)}+\epsilon^{(+)}=0$, the perturbation is marginal at the ordinary and extraordinary fixed points. It is interesting to determine whether the interface remains marginal for any value of $J$, as it happens in the symmetric case. In the truly marginal case (i) the local magnetization exponent is a continuous function of the coupling: $x_{m}^{\text {int }}=x_{m}^{\text {int }}(J)$ [as in Eqs. (14) and (15)] and (ii) the scaling dimension of the local energy-density operator has to remain constant: $x_{e}^{\text {int }}=1$.

\section{NUMERICAL STUDY}

The calculation of the scaling dimensions associated with the interface, $x_{m}^{\text {int }}$ and $x_{e}^{\text {int }}$, is based on a finite-size scaling analysis of the matrix elements of the corresponding operators, as indicated in Eq. (9). The ground state of the system, with a length $L$ for the two subsystems up to 86 (38) for the magnetization (energy) exponent, has been determined using the DMRG method. ${ }^{21}$ In order to obtain a good accuracy, we have generally kept around $m=150$ states of the density matrix.

The magnetization density has been calculated using symmetry-breaking boundary conditions, with the two types of spins held fixed at both ends, $\sigma_{ \pm L}^{z}=\tau_{ \pm L}^{z}=+1$. The magnetization density is determined on both sides of the interface when the system is asymmetric.
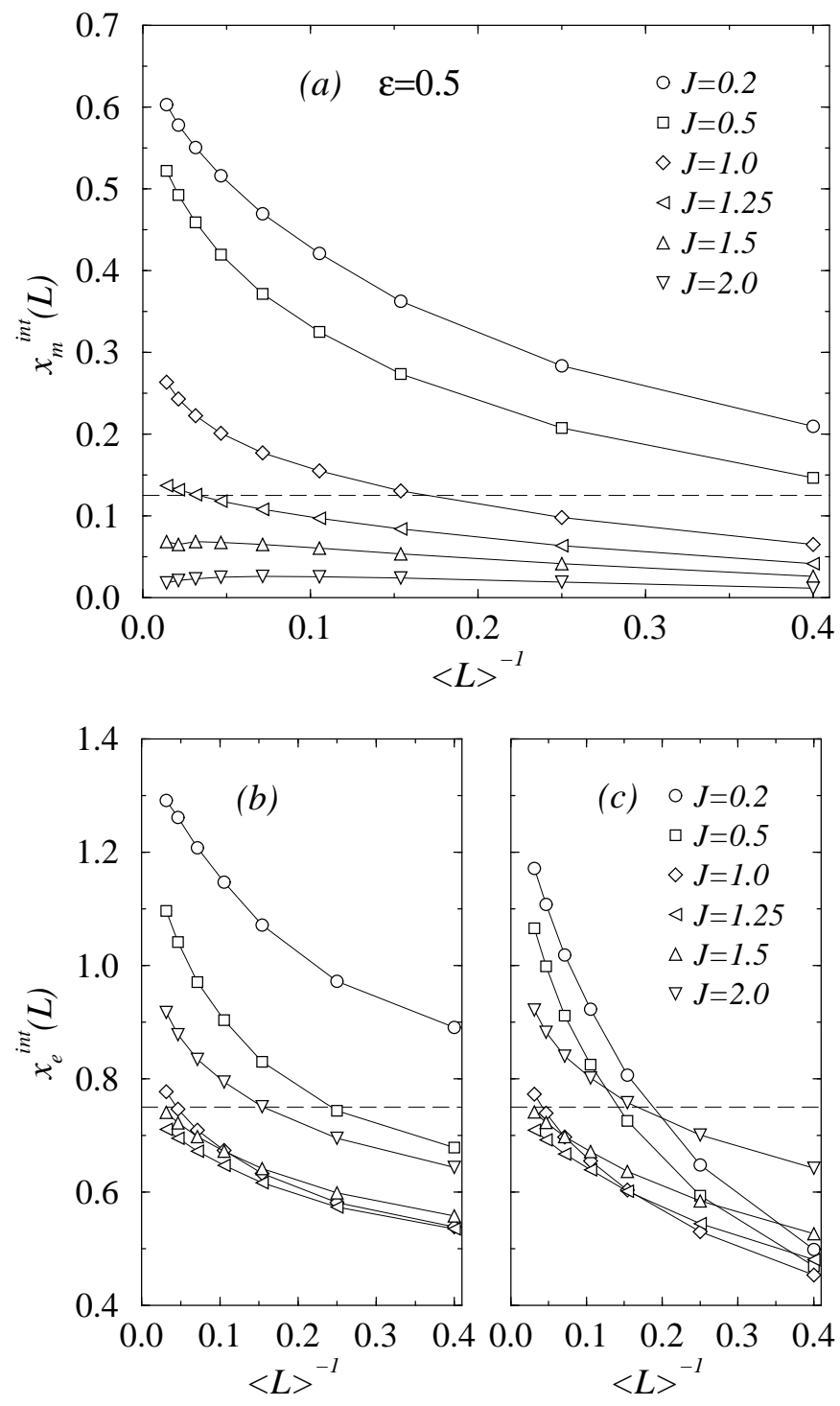

FIG. 3: Interface critical behavior between two identical Ashkin-Teller models with bulk four-spin coupling $\epsilon=0.5$ (relevant case). The upper part gives the magnetization exponents and the lower part the energy-density exponents deduced either from $e_{\mathrm{int}}^{x}$ on one side of the interface (left) or from $e_{\text {int }}^{z}$ on the junction itself (right).

For the energy density, we eliminate the regular contribution to the ground-state expectation value in Eq. (9) by taking the difference of the values obtained for the systems with free and fixed boundary conditions. Since the sign of the singular part generally changes when the boundary conditions are changed, a good precision can be obtained in this way. ${ }^{22}$ As indicated in Eq. (9), we calculate the energy density on the junction itself by taking the ground-state expectation value $\left\langle 0\left|\sigma^{z}(-1) \sigma^{z}(1)\right| 0\right\rangle$ and on both sides of the interface, with $\left\langle 0\left|\sigma^{x}( \pm 1)\right| 0\right\rangle$.

From the values of the singular part of the matrix ele- 

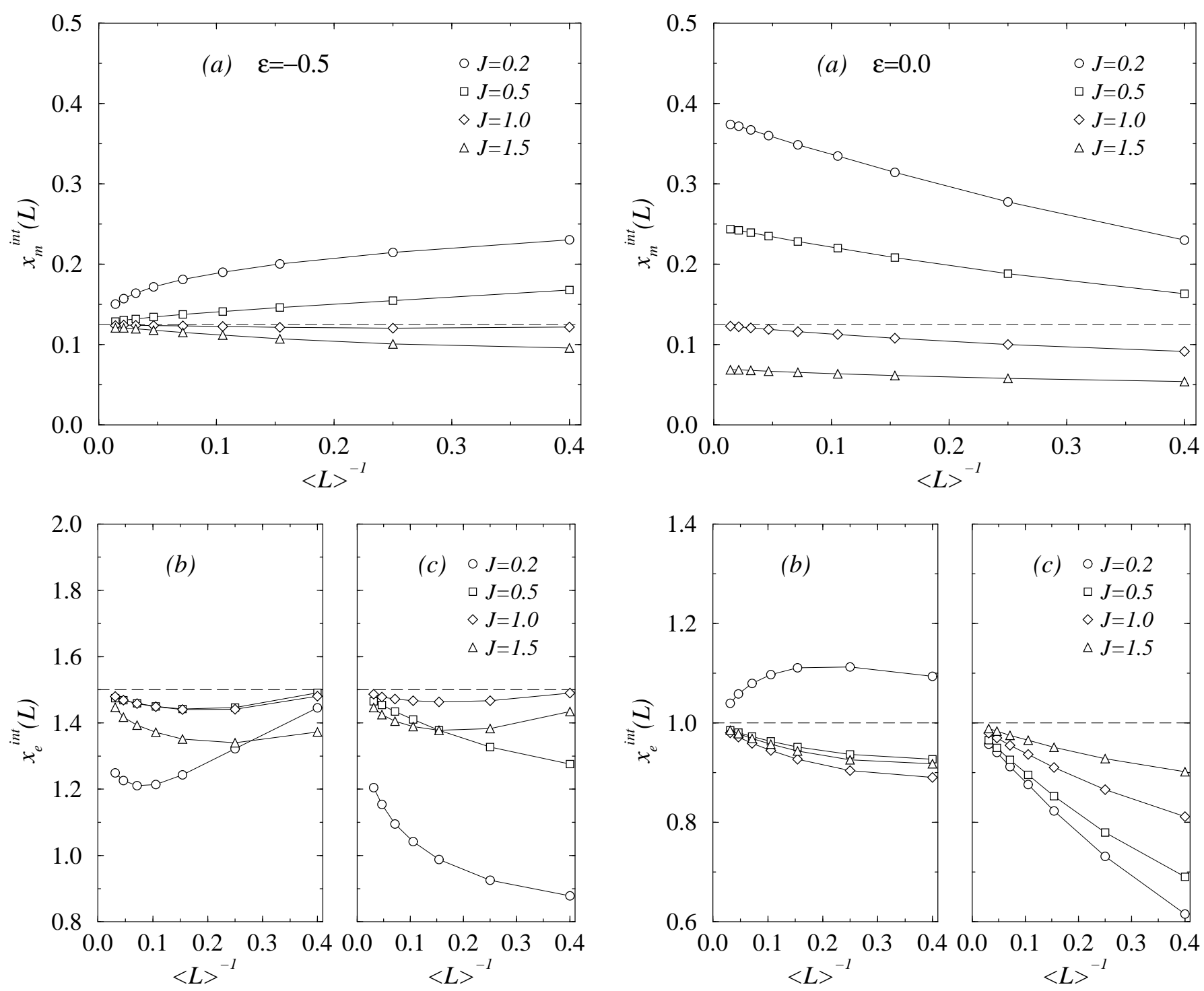

FIG. 4: As in Fig. 3 for the irrelevant case $\epsilon=-0.5$.

ment, say, $m_{\text {int }}(L) \sim L^{-x_{m}^{\text {int }}}$, at two different sizes, $L$ and $b L$, we deduce effective exponents through two-point fits:

$$
\frac{\ln m_{\text {int }}(b L)-\ln m_{\text {int }}(L)}{\ln b}=x_{m}^{\mathrm{int}}(L) .
$$

In order to obtain the same numerical accuracy for the different points, we keep the ratio $b$ between neighboring sizes approximately constant. The effective exponents evolve toward their exact values when the mean size associated with the two-point fit, $\langle L\rangle=L(b+1) / 2$, tends to infinity.

FIG. 5: As in Fig. 3 for the marginal Ising limit $\epsilon=0$.

\section{A. Two identical subsystems}

We first check the validity of the phase diagrams given in Fig. 1 for the interface between identical critical subsystems, the ladder defect in an otherwise homogeneous system. We have studied three values of the bulk fourspin coupling, $\epsilon=0.5,-0.5$, and 0 , and calculated the interface magnetization and energy exponents for different values of the interface coupling $J$. The results are shown in Figs. 3-5.

When $\epsilon=0.5$ (Fig. 3) the perturbation is relevant and the bulk fixed point unstable. For small values of the interface coupling, the flow is toward a free surface behavior. For $J=0.2$, the effective exponents tend to their surface values, $x_{m}^{\text {int }}=x_{m}^{s}=2 / 3$ and either $x_{e}^{\text {int }}=x_{e}^{s}=2$ when the energy operator is the surface energy operator 
of one subsystem $\left(e_{\text {int }}^{x}\right)$ or $x_{e}^{\text {int }}=2 x_{m}^{s}=4 / 3$ when the energy operator involves the surface magnetization operators of the two subsystems $\left(e_{\text {int }}^{z}\right)$. The effective exponents converge slowly to $x_{m}^{\text {int }}=0$ and $x_{e}^{\text {int }}=2$, characteristic of an ordered interface, for the highest values of $J$. The interface exponents take the bulk values, $x_{m}^{\text {int }}=1 / 8$ and $x_{e}^{\text {int }}=3 / 2$, for an intermediate value of $J$, between 1.25 and 1.5 , where the flow is toward the (unstable in the $J$ direction) bulk fixed point.

For $\epsilon=-0.5$ the bulk fixed point is stable and the effective exponents in Fig. 4 approach the bulk values $x_{m}^{\text {int }}=x_{m}=1 / 8$ and $x_{e}^{\text {int }}=x_{e}=3 / 2$, independently of the value of the interface coupling.

The Ising limit $\epsilon=0$ in Fig. 5 is a truly marginal situation. As expected, the interface magnetization exponent is continuously varying with $J$. The extrapolated values are in agreement with the exact results given in Eq. (14): $x_{m}^{\text {int }}(J=0.2)=0.382, x_{m}^{\text {int }}(J=0.5)=0.248$, and $x_{m}^{\text {int }}(J=1.5)=0.07$, respectively. The interface energy exponent takes the bulk value $x_{e}^{\text {int }}=1$, which is necessary for a true marginal behavior at the line defect.

\section{B. Two different subsystems}

For an interface between two different subsystems, we start with the case where $\epsilon^{(-)}+\epsilon^{(+)}>0$, which corresponds to the RG flow in the upper part of Fig. 2,

The results obtained for the magnetization (energy) density exponents when $\epsilon^{(-)}=-0.25$ and $\epsilon^{(+)}=0.5$ are presented in the upper (lower) part of Fig. 6. In accordance with the RG phase diagram, for small $J(J=0.2$ and 0.5$)$, the effective interface exponents slowly approach the surface magnetization exponent of the right subsystem $x_{m}^{s}=2 / 3$, whereas in the other limit $(J=1.5)$ they seem to converge to zero. According to the numerical results, the special interface transition takes place at $J_{s} \approx 1$ where the magnetization exponent is close to $x_{m}=1 / 8$.

The energy-density exponents shown in the lower part of Fig. 6] are greater for small and large values of $J$ than for $J=1.0 \sim J_{s}$. This behavior is expected since $x_{e}^{s}=2$ at the ordinary and extraordinary transitions, whereas the instability of the special interface fixed point requires $x_{e}^{\text {int }}<1$.

When $\epsilon^{(-)}+\epsilon^{(+)}<0$, we are in the situation sketched in the lower part of Fig. 2 2 which was tested for $\epsilon^{(-)}=$ -0.5 and $\epsilon^{(+)}=0.25$. The numerical results are presented in Fig. 7

Here, too, the crossover effects are quite strong for the effective magnetic exponents shown in the upper part of the figure. For a small coupling $J=0.2$, the effective exponents remain close to the surface magnetization exponent of the $\epsilon^{(-)}=-0.5$ model, $x_{m}^{s}=1 / 3$, with a tendency to decrease at the largest sizes. The value of the coupling $J_{s}$ at the special interface fixed point is slightly higher than 1 , where the effective magnetization exponents have the smallest finite-size corrections and
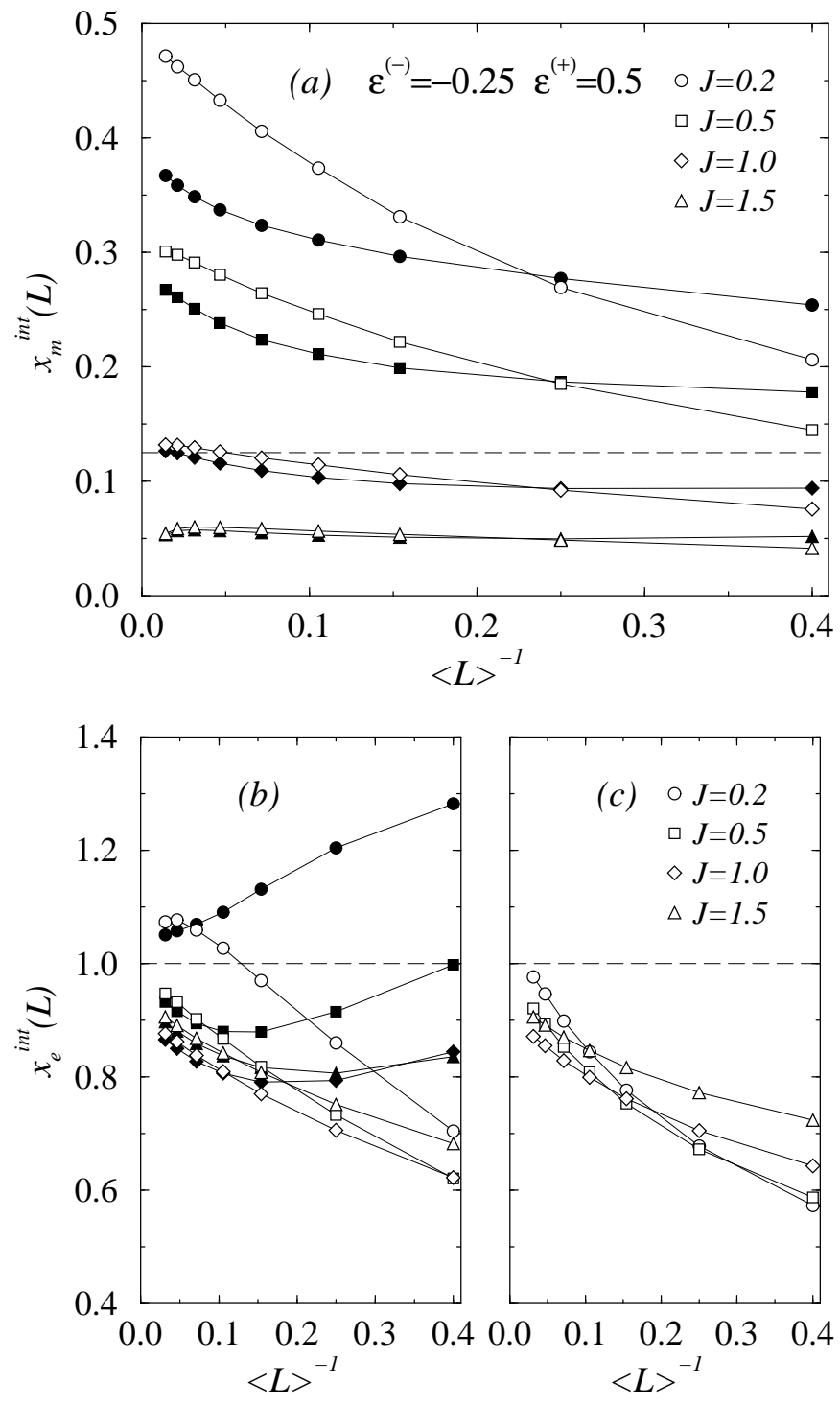

FIG. 6: Interface critical behavior between two Ashkin-Teller models with four-spin couplings $\epsilon^{(-)}=-0.25$ and $\epsilon^{(+)}=$ 0.5 (relevant case). The upper part gives the magnetization exponents calculated either on the left (full symbols) or on the right (open symbols) of the interface. The lower part gives the energy-density exponents deduced from $e_{\text {int }}^{x}$ on the two sides of the interface (left) or from $e_{\mathrm{int}}^{z}$ on the junction itself (right).

the extrapolated value is a little below $x_{m}=1 / 8$.

The stability of the fixed point of the special interface transition is related to the value of the energy-density exponent $x_{e}^{\text {int }}$, which is shown in the lower part of Fig. 7. Except for $J=0.2$, the effective exponents extrapolates to values larger than 1 , in agreement with the stability analysis in Eq. (16). For $J=1.0$, one obtains $x_{e}^{\text {int }}=$ $1.10(2)$, which corresponds to a crossover exponent $y_{i}=$ $-0.10(2)$. This small (negative) value of the crossover exponent explains the slow convergence of the effective 

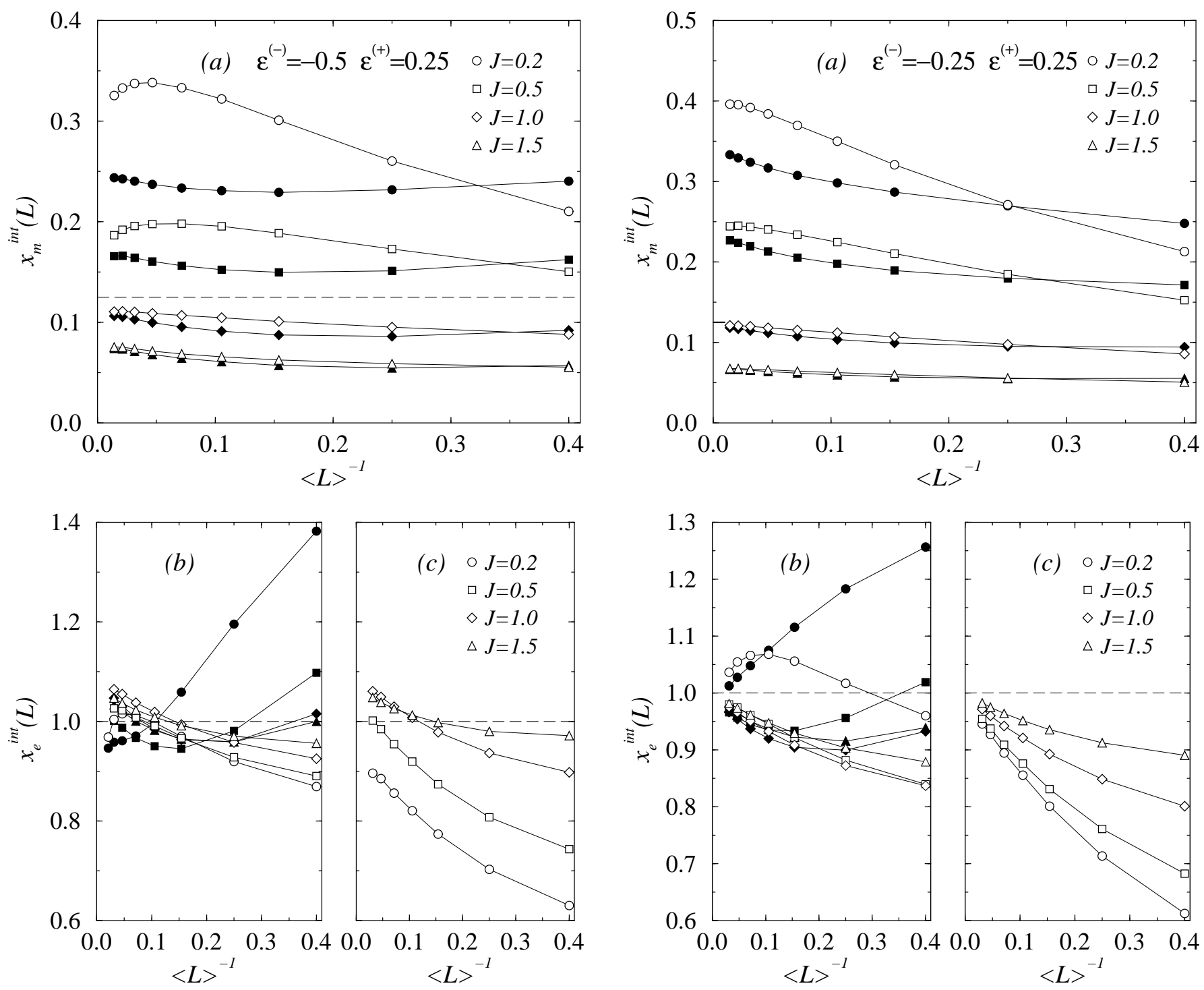

FIG. 7: As in Fig. 6] for $\epsilon^{(-)}=-0.5$ and $\epsilon^{(+)}=0.25$ (irrelevant case).

FIG. 8: As in Fig. 6] for the marginal case with $\epsilon^{(-)}=-0.25$ and $\epsilon^{(+)}=0.25$.

\section{DISCUSSION}

magnetization exponents in the upper part of Fig. 7.

For the marginal situation where $\epsilon^{(-)}+\epsilon^{(+)}=0$, we considered two cases, $\epsilon^{(+)}=0.25$ and 0.5 . The results are shown in Figs. 8 and 9] respectively.

The magnetic exponents seem to vary continuously with $J$, without evidence of crossover effects at large sizes. The possibility that this system is truly marginal is supported by the behavior of the effective energy exponents which, whatever the value of $J$, extrapolate to a value compatible with $x_{e}^{\text {int }}=1$. ior in the AT model is that the local critical exponents are continuously varying with the strength of the junction when the sum of the four-spin couplings vanishes, even in the asymmetric case. Here, we discuss the possible origin of this truly marginal behavior.

We consider a somewhat different setting, where the system is semi-infinite and consists of two subsystems with the shape of corners, $-\infty<z<0,0<y<\infty$ and $0<z<\infty, 0<y<\infty$, connected by a chain junction along the sides at $z=0$. We are interested in the behavior of the generalized corner exponent $x_{m}^{c}$, measured at $y=z=0$. Under a logarithmic conformal mapping, the 

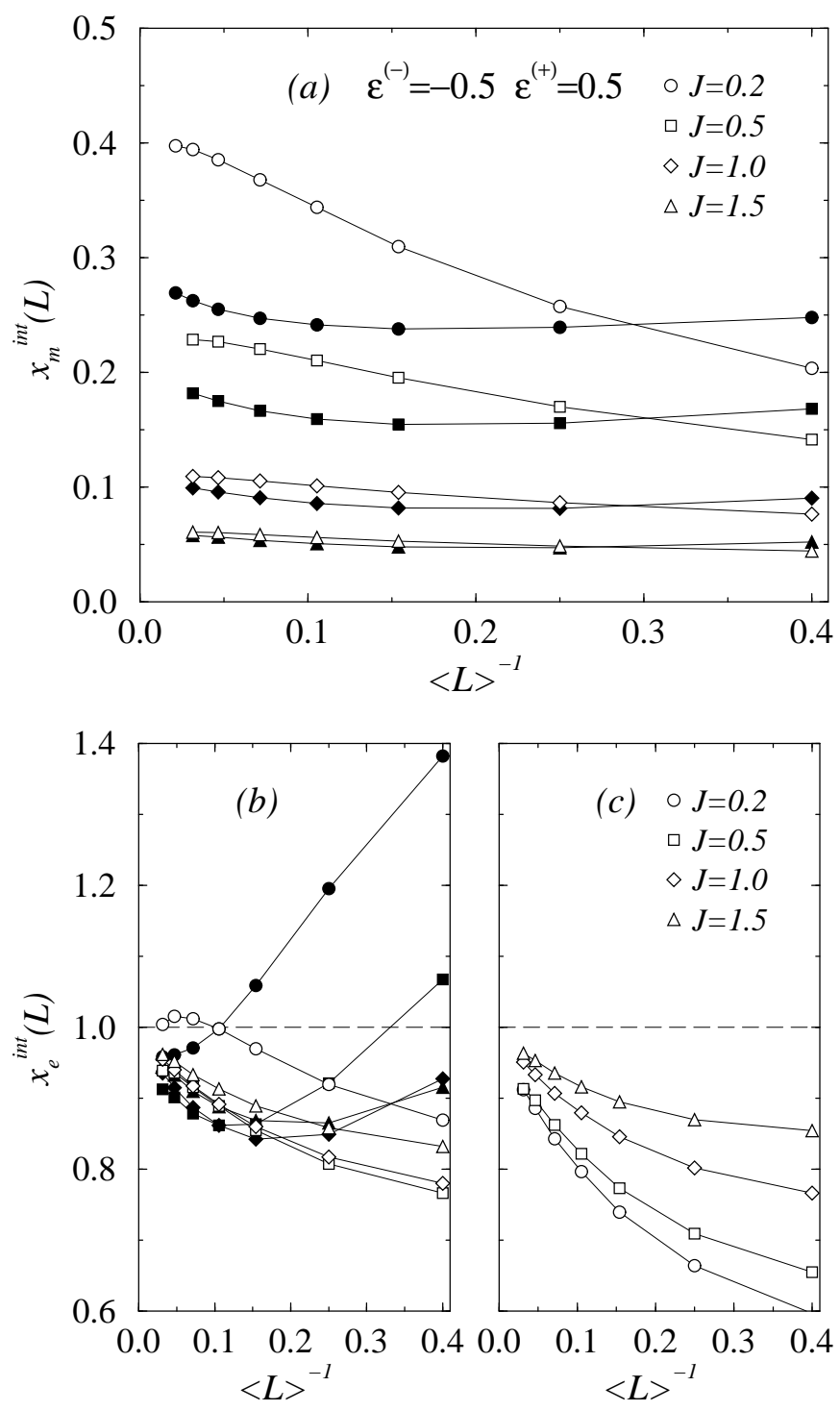

FIG. 9: As in Fig. 6 for the marginal case with $\epsilon^{(-)}=-0.5$ and $\epsilon^{(+)}=0.5$.

critical semi-infinite system is transformed into a strip with open boundaries at $i= \pm L / 2$ and a chain junction at $i=0$. In the Hamiltonian limit, the strip Hamiltonian $\mathcal{H}_{\text {str }}$ involves a transverse field $\widetilde{h}$ at $i=0$, whereas the two ends of the chain are free. In the following, we calculate the first gap $\Delta E(\widetilde{h})$ of $\mathcal{H}_{\text {str }}$, perturbatively for a small transverse field, and deduce the local scaling dimension $x_{m}^{c}$ through the gap-exponent relation of Eq. (10), where $2 \pi$ has to be replaced by $\pi$, the actual angle in the mapping of the semi-infinite system. To calculate the gap, we first perform the duality transformation in Eq. (2). The transformed chain has fixed boundary spins at $i= \pm L / 2$ and a (weak) defect coupling of strength $\widetilde{h}$ at $i=0$. The first gap is given by the difference of the ground-state energies with antiparallel and parallel boundary conditions: $\Delta E(\widetilde{h})=E_{0}^{\uparrow \downarrow}-E_{0}^{\uparrow \uparrow}$. Actually, antiparallel boundary conditions are applied to one type of spin variables, say, $\sigma$, while parallel boundary conditions are always applied to the $\tau$ spin variables. To leading order, only the $\sigma$ spin variables contribute to the difference of the ground-state energies and we obtain

$$
\begin{aligned}
\Delta E(\widetilde{h}) & =2 m_{s}^{(-)}(L / 2) m_{s}^{(+)}(L / 2) \widetilde{h} \\
& =2 a^{(-)} a^{(+)}(L / 2)^{-x_{m}^{(-)}-x_{m}^{(+)} \widetilde{h}}
\end{aligned}
$$

where $m_{s}^{( \pm)}(L / 2)=a^{( \pm)}(L / 2)^{-x_{m}^{( \pm)}}$is the surface magnetization in a critical chain with length $L / 2$, when the spin of the same type on the other end is fixed in the up state. The second-order term vanishes since even contributions to $E_{0}^{\uparrow \uparrow}$ and to $E_{0}^{\uparrow \downarrow}$ are exactly the same. Only odd powers of $\tilde{h}$ are present.

The leading behavior of the gap in Eq. (18) depends on the value of $x_{i}=x_{m}^{(-)}+x_{m}^{(+)}$. For $x_{i}>1$, the first gap vanishes faster than $1 / L$; thus, $x_{m}^{c}=0$ and the junction is ordered. This happens for $\epsilon^{(-)}+\epsilon^{(+)}>0$ and corresponds to the upper part of Fig. 2. On the contrary, for $x_{i}<1$, the gap has a decay slower than $1 / L$; thus, according to Eq. (10), the interface exponent $x_{m}^{\text {int }}$ is formally divergent to leading order of the perturbational calculation. This indicates that the extraordinary interface fixed point with $\widetilde{h}=0$ is unstable, a situation which corresponds to the lower part of Fig. 2, In the marginal case $x_{i}=1$, up to first order in $\widetilde{h}$, the local exponent has the variation

$$
x_{m}^{c}=4 \widetilde{h} \frac{a^{(-)} a^{(+)}}{\pi v_{s}}+O\left(\widetilde{h}^{3}\right),
$$

where the coefficients $a^{( \pm)}$are $O(1)$.

In the Ising limit $\epsilon^{( \pm)}=0, a^{( \pm)}=1$ and, with the parametrization chosen for the quantum Hamiltonian, $v_{s}=2$. Thus we obtain $x_{m}^{c}(\widetilde{h})=\frac{2 \widetilde{h}}{\pi}+O\left(\widetilde{h}^{3}\right)$, which is the leading contribution to the exact result:23 $x_{m}^{c}(\widetilde{h})=1-\frac{2}{\pi} \arctan \left(\widetilde{h}^{-1}\right)$.

In the asymmetric marginal case, $\epsilon^{(-)}+\epsilon^{(+)}=0$, we also have a continuous variation of the leading contribution to $x_{m}^{c}$ with $\widetilde{h}$. We also expect a truly marginal local critical behavior in this case. This assumption is supported by the fact that the second-order term of the expansion is vanishing due to symmetry. In the marginally relevant or irrelevant cases, the second-order term of the expansion is usually diverging as $\log L, 24$ however, for a marginally irrelevant perturbation, the singular terms are expected to sum up to a regular contribution. In our case, we expect a truly marginal behavior and continuously varying local scaling exponents also for the ladder junction studied numerically in Sec. IV]

There are other systems from which similar composite critical systems can be built and for which a truly marginal interface critical behavior could be obtained. Let us mention the 2D $X Y$ model with different temperatures, say, $T^{(-)}$and $T^{(+)}$, both lower than the 
Kosterlitz-Thouless temperature. Another example is the $X X Z$ chain with different anisotropies on the two sides of the junction. One may notice that the AT Hamiltonian can be transformed into a staggered $X X Z$ model through a duality transformation of the $\tau$ spins followed by a duality transformation on all the spins ${ }^{?}$ Finally, let us mention the Potts model in the FortuinKasteleyn representation, for which the number of states $q$ becomes a continuous parameter. Taking two subsystems with $q$ states on one side and $4-q$ states on the other, since $x_{m}^{s}(q)=1-(2 / \pi) \arccos (\sqrt{q} / 2) \stackrel{25}{\stackrel{25}{5} \text { one has }}$ $x_{m}^{s}(q)+x_{m}^{s}(4-q)=1$. It follows that the junction is a marginal perturbation at small coupling. In this case, however, the central charges of the conformal field theory at the two sides of the junction are different; therefore, it needs further investigations to decide if the perturbation remains truly marginal for any coupling strength, as observed in the symmetric Ising limit $q=2$.

\section{Acknowledgments}

This work has been supported by the National Office of Research and Technology under Grant No. ASEP1111, by the Hungarian National Research Fund under Grants No. OTKA TO48721, No. K62588, and No. MO45596. F.I. thanks Université Henri Poincaré for hospitality. The Laboratoire de Physique des Matériaux is Unité Mixte de Recherche CNRS No. 7556.
* Electronic address: turban@lpm.uhp-nancy.fr

$\dagger$ Electronic address: igloi@szfki.hu

${ }^{1}$ K. Binder, in Phase Transitions and Critical Phenomena, edited by C. Domb and J. L. Lebowitz (Academic, London, 1983), Vol. 8, p. 1.

${ }^{2}$ H. W. Diehl, in Phase Transitions and Critical Phenomena, edited by C. Domb and J. L. Lebowitz (Academic, London, 1986), Vol. 10, p. 75.

3 M. Pleimling, J. Phys. A 37, R79 (2004).

${ }^{4}$ B. Berche and L. Turban, J. Phys. A 24, 245 (1991)

5 F. Á. Bagaméry, L. Turban, and F. Iglói, Phys. Rev. B 73, 144419 (2006).

6 J. Ashkin and E. Teller, Phys. Rev. 64, 178 (1943).

7 M. Kohmoto, M. den Nijs, and L.P. Kadanoff, Phys. Rev. B 24, 5229 (1981).

${ }^{8}$ F. Iglói and J. Sólyom, J. Phys. A 17, 1531 (1984).

${ }^{9}$ C. Fan, Phys. Lett. 39A, 136 (1972).

10 J. L. Cardy, in Phase Transitions and Critical Phenomena, edited by C. Domb and J. L. Lebowitz (Academic, London, 1987), Vol. 11.

11 B. Nienhuis, in Phase Transitions and Critical Phenomena, edited by C. Domb and J. L. Lebowitz (Academic, London, 1987), Vol. 11.

12 M. Baake, G. von Gehlen, and V. Rittenberg, J. Phys. A 20, L479 (1987).

13 G. von Gehlen and V. Rittenberg, J. Phys. A 20, 227 (1987).
14 R. Z. Bariev, Zh. Eksp. Teor. Fiz. 77, 1217 (1979) [Sov. Phys. JETP 50, 613 (1979)].

15 F. Iglói, I. Peschel, and L. Turban, Adv. Phys. 42, 683 (1993).

16 J. L. Cardy, J. Phys. A 17, L385 (1984).

17 T. W. Burkhardt, in Lecture Notes in Physics, Proceedings of the XXth Winter School, Karpacz, Poland, 1984, edited by A. Pekalski and J. Sznajd (Springer, Berlin, 1984), Vol. 206, p. 169.

18 T. W. Burkhardt and E. Eisenriegler, Phys. Rev. B 24, 1236 (1981).

19 H. W. Diehl, S. Dietrich, and E. Eisenriegler, Phys. Rev. B 27, 2937 (1983).

20 E. Eisenriegler and T. W. Burkhardt, Phys. Rev. B 25, 3283 (1982).

21 For recent reviews about the DMRG method, see U. Schollwock, Rev. Mod. Phys. 77, 259 (2005); K. Hallberg, Adv. Phys. 55, 477 (2006).

22 D. Karevski, P. Lajkó, and L. Turban, J. Stat. Phys. 86, 1153 (1996).

23 M. Henkel, A. Patkós, and M. Schlottmann, Nucl. Phys. B 314, 609 (1989).

${ }^{24}$ F. Iglói, L. Turban, and B. Berche, J. Phys. A 24, L1031 (1991).

25 J. L. Cardy, Nucl. Phys. B 240, 514 (1984). 